\title{
A Prostacycline Analog Prevents Chronic Myocardial Remodeling in Murine Cardiac Allografts
}

\author{
Jun-ichi Suzuki, ${ }^{1}$ MD, Masahito Ogawa, ${ }^{1}$ MS, Yoshiki SAKaI, ${ }^{2} \mathrm{PhD}$, Yasunobu Hirata, ${ }^{1}$ MD, \\ Mitsuaki Isobe, ${ }^{3} \mathrm{MD}$, and Ryozo NAGAI, ${ }^{4} \mathrm{MD}$
}

\begin{abstract}
SUMMARY
ONO-1301MS is a compound that acts as a prostacyclin agonist with thromboxane A2 synthase inhibitory activity. We investigated the effect of ONO-1301MS on myocardial remodeling in murine cardiac allografts. The hearts of Balb/c mice were transplanted into $\mathrm{C} 3 \mathrm{H} / \mathrm{He}$ mice (a full allomismatch combination) to assess acute rejection or $\mathrm{C} 57 \mathrm{BL} / 6$ hearts into B6.C-H2 ${ }^{<m 12>} \mathrm{KhEg}$ (a class II mismatch combination) to examine chronic rejection. ONO-1301MS did not prolong full allomismatch cardiac graft survival. Severe myocardial fibrosis with high collagen concentration was observed in untreated class II mismatch allografts on day 60 . However, significantly suppressed myocardial fibrosis with less collagen synthesis was observed in the ONO-1301MS-treated group compared to the control group. ONO-1301MS could be an effective strategy to suppress chronic myocardial remodeling in cardiac transplantation. (Int Heart J 2012; 53: 64-67)
\end{abstract}

Key words: Prostacyclin, Collagen, Inflammation, Transplantation

$\mathrm{C}$ nical cardiac transplantation has been developed as a therapy for end-stage congestive heart failure. ${ }^{1)}$ Several therapeutic trials aimed at preventing myocardial remodeling induced by chronic rejection have been performed, although none have had significant success at this point. ${ }^{2)}$ Prostacycline, a potent vasodilator and inhibitor of platelet aggregation, is a major product of the cyclooxygenase pathway. There are several reports that describe the anti-inflammatory effect of prostacyclin using prostacyclin receptor (IP)-deficient mice. ${ }^{3,4)}$ It has been reported that the prostacyclin analog elevated Th2 cytokines, altering the function of dendritic cells. ${ }^{5)}$ Recently, we developed a new prostacyclin agonist, ONO-1301MS, which has long-lasting prostacyclin activity and an inhibitory effect on thromboxane synthase. ${ }^{6)}$ Because ONO-1301MS does not have prostanoid structures, it is not easily metabolized and its effects remain for a longer time compared with other prostacyclin analogs, such as beraprost or iloprost. ${ }^{7-10)}$ It has been reported that local administration of a slow releasing form of ONO-1301MS into ischemic murine hearts was effective at preventing left ventricular remodeling and improving the survival rate. ${ }^{11)}$ It was also reported that local delivery of a slow releasing form of ONO-1301MS augmented collateral growth and improved cardiac function in a swine chronic cardiac ischemia model. ${ }^{12)}$

Here, we investigated therapeutic efficacy of ONO$1301 \mathrm{MS}$ in murine heterotopic cardiac transplantation models, evaluating acute and chronic rejection. Our findings suggest that ONO-1301MS is effective in the prevention of myocardial remodeling induced by chronic rejection.

\section{Methods}

Compound: The novel prostacyclin selective agonist ONO1301MS was kindly provided by Ono Pharmaceutical Co. Ltd. The regimen used in the experiments was determined from those used in previous papers. ${ }^{8-12)}$

Animals and cardiac transplantation: Male inbred mice (4 to 6 weeks, 20-25 g) were used. The male BALB/c $\left(\mathrm{H}-2^{\mathrm{d}}\right)$ and C57BL/6 (H-2 ${ }^{\mathrm{b}}$ ) mice (4-6 weeks, 20-25 g) were obtained from Japan Clea, Co (Tokyo), and this combination was used as the major histocompatibility complex total allomismatch group for analysis of acute rejection. The C57BL/6 (B/6, H-2 $\left.{ }^{\mathrm{b}}\right)$ and B6.C-H-2 $2^{\text {bm12 }} \mathrm{KhEg}\left(\mathrm{Bm} 12, \mathrm{H}-2^{\mathrm{bm} 12}\right.$ ) combination was used as the class II mismatch combination for analyzing chronic rejection indicated by pathological findings such as ventricular fibrosis and remodeling. Isografts (B/6 to $\mathrm{B} / 6)$ were used as negative controls. They were anesthetized by intraperitoneal injection of $3.6 \%$ hydrochloride $(0.2 \mathrm{~mL} / 20 \mathrm{~g}$, Wako Pure Chemical Industries, Ltd., Osaka, Japan) as reported previously. Allografts were heterotopically transplanted in an intra-abdominal location using a microsurgical technique. Briefly, this technique involved anastomosing the end of the donor aorta to the side of the recipient abdominal aorta, after which the donor pulmonary artery was connected to the inferior vena cava of the recipient mice to return myocardial blood flow. Ischemic time averaged 60 minutes, and the overall success rate was greater than $90 \%{ }^{13-16)}$ This investigation conformed to the Guides for the Care and Use of Laboratory Animals of Tokyo Medical and Dental University and The University of To-

From the ${ }^{1}$ Department of Advanced Clinical Science and Therapeutics, The University of Tokyo, ${ }^{2}$ Ono Pharmaceutical Co. Ltd, ${ }^{3}$ Department of Cardiovascular Medicine, Tokyo Medical and Dental University, and ${ }^{4}$ Department of Cardiovascular Medicine, The University of Tokyo, Tokyo, Japan.

This study was supported by grants from the Research Foundation for Pharmaceutical Sciences, Fujio Mitsui Foundation, and the Japan Society for the Promotion of Science (JSPS) through its "Funding Program for World-Leading Innovative R\&D on Science and Technology (FIRST Program)".

Address for correspondence: Jun-ichi Suzuki, MD, Department of Advanced Clinical Science and Therapeutics, The University of Tokyo, 7-3-1 Hongo, Bunkyo-ku, Tokyo 113-8655, Japan.

Received for publication September 5, 2011

Revised and accepted November 10, 2011. 
kyo and to NHI Guidelines.

Treatment protocol: The recipients of acute rejection models (a full allomismatch combination) were assigned randomly into treatment and vehicle groups. The treatment group mice in the acute rejection model were subcutaneously administered ONO-1301MS $(200 \mathrm{mg} / \mathrm{kg})$ before the transplant operation. Graft survival and function was evaluated by daily palpation, and cessation of beating was interpreted as rejection. The recipients of chronic rejection models were also assigned randomly into treatment and vehicle groups. The treatment group mice in the chronic model (a class II allomismatch) were subcutaneously administered ONO-1301MS (200 mg/kg) every 3 weeks after transplantation. Cardiac grafts in the chronic model were harvested immediately after the mice were sacrificed on day 60 .

Histopathology: The grafts were harvested and sectioned transversely at the maximal circumference of the ventricle. Serial sections $(6 \mu \mathrm{m})$ from tissue embedded in OCT were stained with hematoxylin and eosin (HE) and Mallory staining. The grafts were photographed and processed using an image analysis system to calculate the area of cell infiltration and fibrosis. The areas of myocardial cell infiltration using HE staining and fibrosis using Mallory staining were determined with a computer-assisted analyzer (Scion Image beta 4.0.2). The area ratio was calculated as (affected area $\times 100) /$ whole area $(\%)$. Values for three ventricular regions were averaged for each heart, and the mean percentage of affected area for each group was calculated as described. ${ }^{13-16)}$

Collagen content evaluation by hydroxyproline assay: The myocardial collagen content in the allografts was measured by hy-

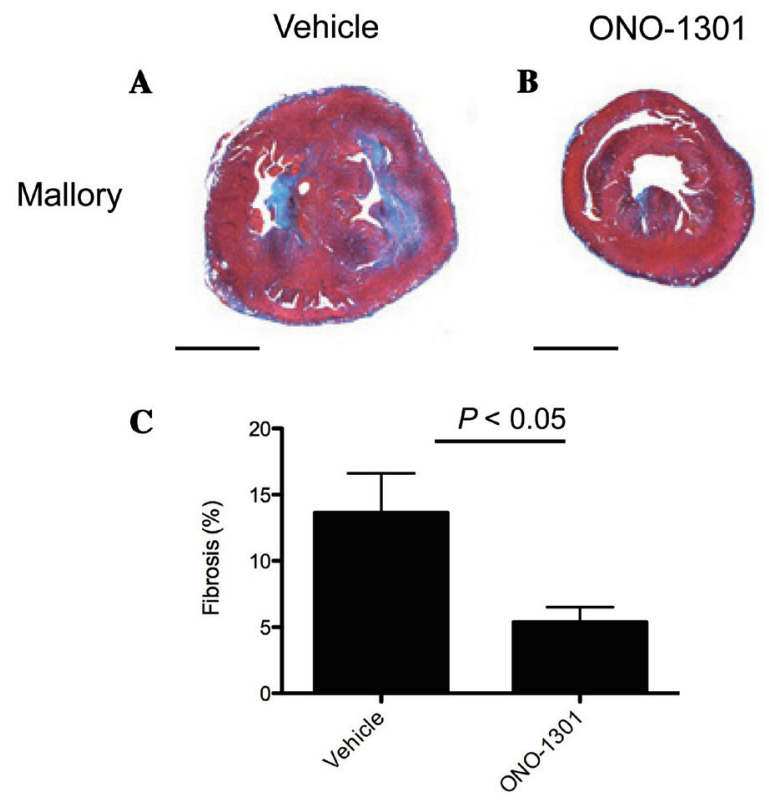

Figure 1. Histological findings of the myocardium. Panels $\mathbf{A}$ and $\mathbf{B}$ show representative pathological results with Mallory staining of the class II mismatch allografts on day 60. Panel $\mathbf{C}$ shows the quantitative data of fibrosis per whole area. Severe fibrosis was observed in the nontreated allografts on day 60 (A). However, ONO-1301MS markedly attenuated myocardial fibrosis (B). The fibrosis area ratio of the ONO-1301MS group was statistically less than that of the vehicle group $(\mathbf{C})$. droxyproline assay as previously reported. ${ }^{17)}$ Briefly, each sample was homogenized in acetic buffer and hydrolyzed for 24 hours at $100^{\circ} \mathrm{C}$, followed by neutralization with $6 \mathrm{~mol} / \mathrm{L} \mathrm{NaOH}$. Chloramine-T reagent and Ehrlich's reagent solution were added, and the samples were mixed and incubated at $60^{\circ} \mathrm{C}$ for 30 minutes. The absorbency of the samples was read at $560 \mathrm{~nm}$, and the hydroxyproline concentration was assessed with a curve obtained from hydroxyproline standard solutions. Data are expressed as micrograms of collagen per gram dry weight.

Real-time PCR: Total RNA was isolated from each individual heart with TRIzol reagent (Invitrogen/Life Technologies, San Diego, CA) after homogenization with a polytron homogenizer. Real-time PCR was used to determine the mRNA expression of interferon (IFN)-gamma (Assay ID: Mm01168134_m1), interleukin (IL)-2 (Assay ID: Mm00434256_m1), IL-10 (Assay ID: Mm00439614_m1), matrix metalloproteinase (MMP)-2 (Assay ID: Mm00439498_m1) and MMP-9 (Assay ID: Mm00442991_m1) in the grafts. The cDNA was run in duplicates. To account for differences in cDNA preparation and cDNA amplification efficiency, the mRNA expression of each target gene was normalized by GAPDH (Assay ID: Mm 99999915_g1). Quantitative data were calculated using the comparative CT $(\Delta \Delta \mathrm{CT})$ method. ${ }^{16)}$

Statistical analysis: All data are expressed as the mean \pm SEM. Graft survival analysis was performed using the Kaplan-Meier method with the log-rank test. Data were compared, and differences between the two groups were analyzed using the $t$-test. Differences with values of $P<0.05$ were considered significant.

\section{RESULTS}

Graft survival: ONO-1301MS did not statistically prolong graft survival in the full-allomismatch combination of C57BL/6 (H-2b) to BALB/c (H-2d) (data not shown).

Histological findings in the myocardium: Isografts and allografts from the class II mismatch group kept beating through-

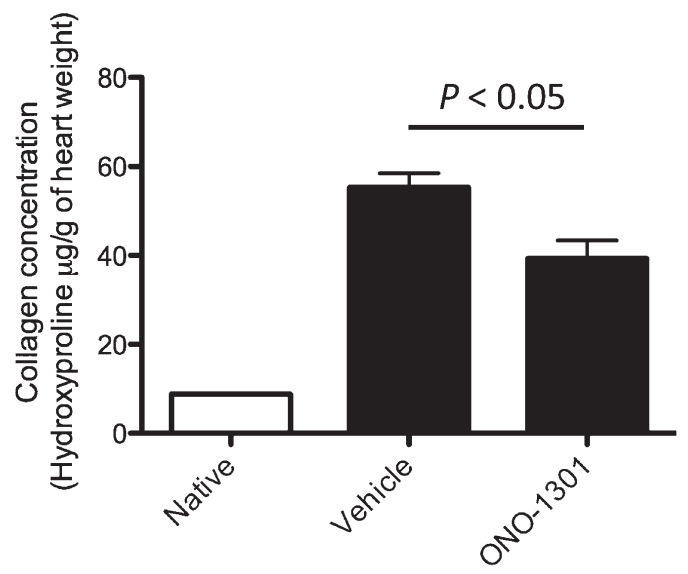

Figure 2. Collagen content in the allografts. The effect of ONO-1301MS treatment on collagen network stabilization was evaluated using a biochemical quantification assay. The collagen content in the allografts was significantly lower in the ONO-1301MS-treated group than in the vehicletreated control group. 
$\mathbf{A}$

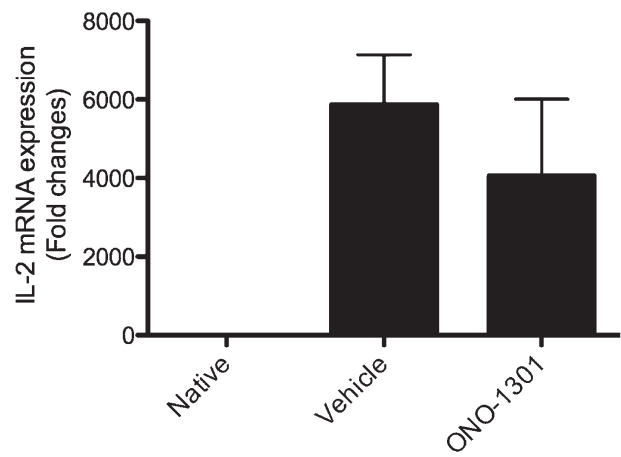

B

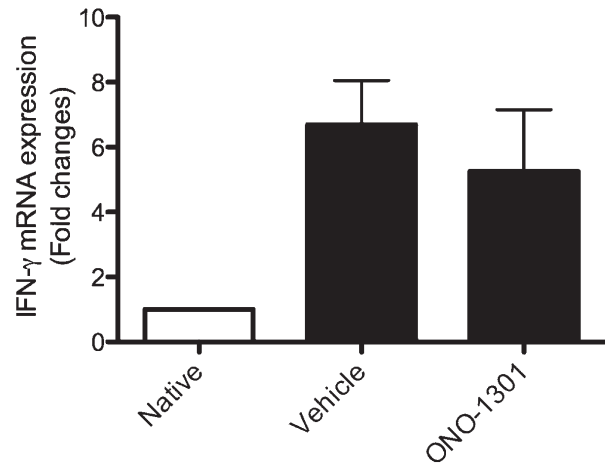

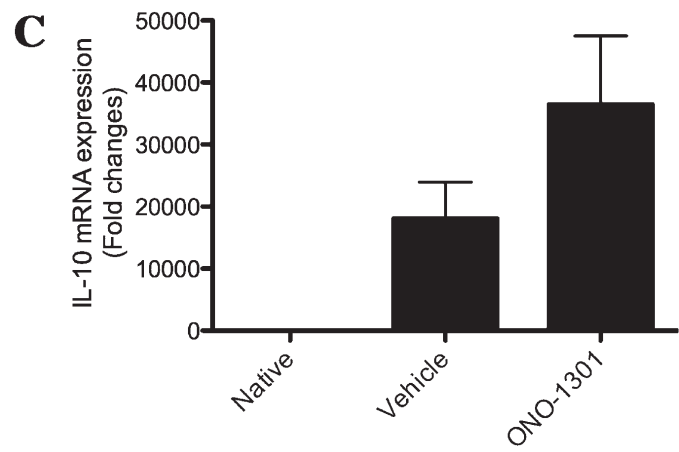

Figure 3. Cytokine mRNA expression. Real-time RTPCR was used to examine expression of cytokine mRNA in allografts. These cytokine transcription levels were statistically comparable between the two groups. However, the mRNA levels of IL-2 (A) and IFN-gamma (B) were slightly suppressed while the mRNA level of IL-10 (C) was slightly elevated in the ONO-1301MS treated group compared with that of the vehicle-treated group $(n=4$ each $)$
$\mathbf{A}$

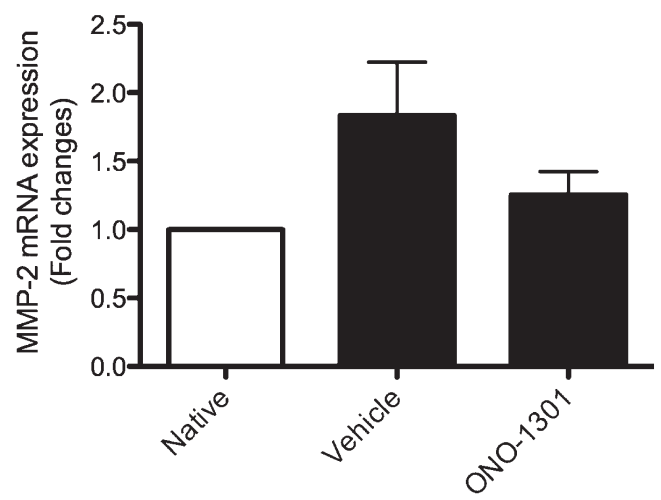

B

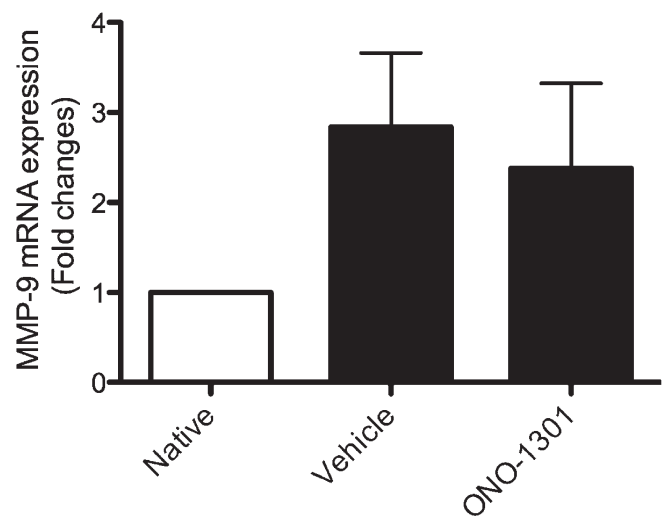

Figure 4. MMP mRNA expression. Because gelatinases play a key role in collagen synthesis, we chose MMP-2 and MMP-9 to analyze this mechanism. Although these transcription levels were statistically comparable between the two groups, the mRNA levels of MMP-2 (A) and MMP-9 (B) were slightly suppressed in the ONO-1301MS treated group compared with that of the vehicle-treated group ( $n=4$ each). out the observation period. In the class II mismatch group, severe myocardial fibrosis was observed in nontreated allografts on day 60. However, ONO-1301MS markedly attenuated myocardial fibrosis. The fibrosis score of the ONO-1301MS group was statistically less than the vehicle group (Figure 1).

Collagen content was suppressed in the ONO-1301MS-treated hearts: The effect of ONO-1301MS treatment on collagen network stabilization was evaluated by the biochemical quantification assay. The collagen content in the allografts was significantly lower in the ONO-1301MS-treated group than the vehicle-treated group (Figure 2).

Cytokine mRNA expression: Real-time RT-PCR was used to examine the expression of cytokine mRNA in allografts. Because previous papers indicated that IL-2 and IFN-gamma are critical Th 1 cytokines and IL-10 is a key Th 2 cytokine in transplantation, we chose them to analyze this mechanism. The cytokine transcription levels were statistically comparable between the two groups. However, mRNA levels of IL-2 and IFN-gamma were slightly suppressed and those of IL-10 were slightly elevated in the ONO-1301MS-treated group compared with that of the non-treated group $(n=4$ each, Figure 3$)$.

MMP mRNA expression: Since gelatinases play a key role in collagen synthesis, we chose MMP-2 and MMP-9 to analyze this mechanism. Although these transcription levels were statistically comparable between the two groups, the mRNA levels of MMP-2 and MMP-9 were slightly suppressed in the ONO-1301MS-treated group compared with the vehicle-treated group ( $n=4$ each, Figure 4$)$.

\section{Discussion}

Prostacyclin is a known major regulator of inflammation and a deficiency in its receptors alters inflammation. It was reported that prostacyclin analogs had an important role in the inhibition of Th1 and Th2 cytokine production from CD4 posi- 
tive T cells. Prostacyclin analogs inhibit the production of multiple proinflammatory cytokines and chemokines, and antigenspecific activation of CD4 positive T cells. These modulatory effects appear to be mediated through NF-kB signaling pathways, because prostacyclin analogs decrease NF-kB activity. ${ }^{18)}$ It is well known that proinflammatory cytokines, chemokines, and NF-kB play a critical role in the development of cardiac rejection. Therefore, we attempted to clarify the prostacyclinrelated mechanisms of rejection using a novel prostacyclin agonist ONO-1301MS and murine cardiac transplantation models.

In this study, ONO-1301MS reduced the myocardial collagen concentration that resulted in suppressed myocardial remodeling induced by chronic rejection. Previously, it was reported that prostacyclin activation suppressed NF-kB activity ${ }^{18)}$ that regulated cytokines and MMPs in cardiac transplantation. ${ }^{19)}$ It is also known that cytokines and MMPs play a pivotal role in collagen synthesis and fibrotic myocardial remodeling. ${ }^{20)}$ Our data indicate that ONO-1301MS slightly decreases these proinflammatory cytokines and MMPs, resulting in reduced fibrosis and collagen content in chronically rejected cardiac allografts.

Administration of a high amount of ONO-1301 caused hypotension in rodent models (Sakai, Y. unpublished). To avoid possible adverse effects, we developed ONO-1301MS, which is a slow-releasing form of ONO-1301. In this study, ONO-1301MS did not induce any adverse effect, including hypotension, during the long-term observation period (60 days) in mice. Other papers also demonstrated that ONO$1301 \mathrm{MS}$ was not only effective but also safe in various animal models. ${ }^{12,21,22)}$ At this point, we have some unpublished data from human and monkey experiments. These preliminary results suggest that ONO-1301MS can be clinically used without severe systemic adverse effects.

In summary, ONO-1301MS prevented myocardial remodeling induced by chronic cardiac rejection through suppression of collagen concentration. The novel selective prostacyclin agonist ONO-1301MS may be a new strategy for suppressing not only chronic myocardial remodeling but also other collagen-induced cardiovascular diseases.

\section{ACKNOWLEDGMENTS}

We thank Ms. Noriko Tamura and Ms. Yasuko Matsuda for their excellent technical assistance.

\section{REFERENCES}

1. Stehlik J, Edwards LB, Kucheryavaya AY, et al. The Registry of the International Society for Heart and Lung Transplantation: twenty-seventh official adult heart transplant report--2010. J Heart Lung Transplant 2010; 29: 1089-103.

2. Suzuki J, Isobe M, Morishita R, Nagai R. Characteristics of chronic rejection in heart transplantation: important elements of pathogenesis and future treatments. Circ J 2010; 74: 233-9. (Review)

3. Takahashi Y, Tokuoka S, Masuda T, et al. Augmentation of allergic inflammation in prostanoid IP receptor deficient mice. Br J Pharmacol 2002; 137: 315-22.
4. Nagao K, Tanaka H, Komai M, Masuda T, Narumiya S, Nagai H. Role of prostaglandin $\mathrm{I} 2$ in airway remodeling induced by repeated allergen challenge in mice. Am J Respir Cell Mol Biol 2003; 29: 314-20.

5. Idzko M, Hammad H, van Nimwegen M, et al. Inhaled iloprost suppresses the cardinal features of asthma via inhibition of airway dendritic cell function. J Clin Invest 2007; 117: 464-72.

6. Kataoka M, Nagaya N, Satoh T, et al. A long-acting prostacyclin agonist with thromboxane inhibitory activity for pulmonary hypertension. Am J Respir Crit Care Med 2005; 172: 1575-80.

7. Imawaka H, Sugiyama Y. Kinetic study of the hepatobiliary transport of a new prostaglandin receptor agonist. J Pharmacol Exp Ther 1998; 284: 949-57.

8. Hayashi K, Nagamatsu T, Oka T, Suzuki Y. Modulation of antiglomerular basement membrane nephritis in rats by ONO-1301, a non-prostanoid prostaglandin I 2 mimetic compound with inhibitory activity against thromboxane A2 synthase. Jpn J Pharmacol 1997; 73: 73-82.

9. Rudd JA, Qian Y, Tsui KK, Jones RL. Non-prostanoid prostacyclin mimetics as neuronal stimulants in the rat: comparison of vagus nerve and NANC innervation of the colon. Br J Pharmacol 2000; 129: 782-90.

10. Murakami S, Nagaya N, Itoh T, et al. Prostacycline agonist with thromboxane synthase inhibitory activity (ONO-1301) attenuates bleomycin-induced pulmonary fibrosis in mice. Am J Physiol Lung Cell Mol Physiol 2006; 290: L59-65.

11. Nakamura K, Sata M, Iwata H, et al. A synthetic small molecule, ONO-1301, enhances endogenous growth factor expression and augments angiogenesis in the ischaemic heart. Clin Sci (Lond) 2007; 112: 607-16.

12. Iwata H, Nakamura K, Sumi M, et al. Local delivery of synthetic prostacycline agonist augments collateral growth and improves cardiac function in a swine chronic cardiac ischemia model. Life Sci 2009; 85: 255-61.

13. Suzuki J, Isobe M, Morishita R, et al. Prevention of graft coronary arteriosclerosis by antisense cdk2 kinase oligonucleotide. Nat Med 1997; 3: 900-3.

14. Suzuki J, Ogawa M, Sagesaka YM, Isobe M. Tea catechins attenuate ventricular remodeling and graft arterial diseases in murine cardiac allografts. Cardiovasc Res 2006; 69: 272-9.

15. Suzuki J, Koga N, Kosuge H, et al. Pitavastatin suppresses acute and chronic rejection in murine cardiac allografts. Transplantation 2007; 83: 1093-7.

16. Ogawa M, Suzuki J, Hishikari K, Takayama K, Tanaka H, Isobe M. Clarithromycin attenuates acute and chronic rejection via matrix metalloproteinase suppression in murine cardiac transplantation. J Am Coll Cardiol 2008; 51: 1977-85.

17. Stegemann H, Stalder K. Determination of hydroxyproline. Clin Chim Acta 1967; 18: 267-73. (Review)

18. Zhou W, Hashimoto K, Goleniewska K, et al. Prostaglandin I2 analogs inhibit proinflammatory cytokine production and $\mathrm{T}$ cell stimulatory function of dendritic cells. J Immunol 2007; 178: 70210.

19. Suzuki J, Morishita R, Amano J, Kaneda Y, Isobe M. Decoy against nuclear factor-kappa B attenuates myocardial cell infiltration and arterial neointimal formation in murine cardiac allografts. Gene Ther 2000; 7: 1847-52.

20. Wei L. Immunological aspect of cardiac remodeling: T lymphocyte subsets in inflammation-mediated cardiac fibrosis. Exp Mol Pathol 2011; 90: 74-8. (Review)

21. Hirata Y, Soeki T, Akaike M, Sakai Y, Igarashi T, Sata M. Synthetic prostacycline agonist, ONO-1301, ameliorates left ventricular dysfunction and cardiac fibrosis in cardiomyopathic hamsters. Biomed Pharmacother 2009; 63: 781-6.

22. Obata H, Sakai Y, Ohnishi S, et al. Single injection of a sustainedrelease prostacyclin analog improves pulmonary hypertension in rats. Am J Respir Crit Care Med 2008; 177: 195-201. 